\title{
Hepatoblastoma requiring liver transplantation. A case report
}

\author{
Roxana-Cristina Mares ${ }^{1}$, Mihaela Chincesan ${ }^{1}$, Emoke Horvath ${ }^{2}$, Horea Gozar ${ }^{3}$, \\ Cristina Oana Marginean ${ }^{1}$ \\ ${ }^{1}$ Department of Pediatrics, University of Medicine, Pharmacy, \\ Sciences and Technology Tg. Mures, Romania \\ 2Department of Pathology, University of Medicine, Pharmacy, \\ Sciences and Technology Tg. Mures, Romania \\ ${ }^{3}$ Department of Pediatric Surgery, University of Medicine, Pharmacy, \\ Sciences and Technology Tg. Mures, Romania
}

\begin{abstract}
Hepatoblastoma is a rare pathology in pediatric patients, occurring in 5-10/one million children. A two year old patient was admitted to our clinic for intense abdominal pain. On examination he presented a large mass in the right hypochondrium. Abdominal ultrasound and computer tomography (CT) revealed a large, solid hepatic tumor with thrombosis of the right portal vein. Serum alpha fetoprotein (AFP) level and thrombocyte number were increased. Liver biopsy was performed and the histopathological aspect was specific for an epithelial hepatoblastoma. Chemotherapy was initiated, followed by surgery in two steps: right portal branch ligation and subsequent tumor resection. Six months after surgery a relapse was suspected, confirmed by CT and elevated AFP levels. A ,rescue" liver transplantation was performed and the patient is disease-free for three years.

Elevated AFP and thrombocyte levels point to the diagnosis of hepatoblastoma. Liver transplantation is the final option for extensive, inoperable or relapsing tumors.
\end{abstract}

Keywords: hepatoblastoma, chemotherapy, relapse, liver transplantation

\section{Abbreviation list}

CT: Computer tomography

AFP: Alpha-fetoprotein

Hgb: Haemoglobin

AST: Aspartate-aminotranspherase

ALT: Alanine-aminotranspherase

\author{
LDH: lactate dehydrogenase \\ CRP: C-reactive protein \\ ESR: erythrocyte sedimentation rate \\ HIV: Human immunodeficiency virus
}

\section{INTRODUCTION}

Hepatoblastoma is a rare malignancy, accounting for just over $1 \%$ of total pediatric cancers (1) and occurs in 5-10/one million children per year (2).

The etiology of the disease is unknown. Several genetic alterations have been found to be associated with hepatoblastoma, the most common are trisomy 2, 8 and 20 (3). Whole-genome DNA analysis led to the detection of many altered genomic regions, of which gain of material on chromosome $2 q$ $13-22,2 q 36-37$ and deletions of $2 p$ and $4 q$ were associated with advanced tumors and poor progno$\operatorname{sis}(3,4)$.
The diagnosis of hepatoblastoma is suspected in the patient aged between 6 months and 3 years, in the presence of a hepatic tumor, thrombocytosis and a high level of serum alpha-fetoprotein (AFP) (3). A high serum level of AFP should be interpreted according to age, very high values indicating hepatoblastoma. Moderately high values of serum AFP can be detected in yolk sack tumors, hepatocarcinoma as well as in certain benign tumors (mesenchymal hamartoma, focal nodular hyperplasia and infantile hemangioendothelioma)(4).

The hepatoblastoma histological classification comprises of six types grouped into two large categories: 1) epithelial hepatoblastoma and 2) epithe- 
lial and mesenchymal mixed hepatoblastoma. Pure epithelial tumors account for approximately 56\% of cases; they contain varying amounts of fetal cells, embryonal cells, or both. Within this group, purely fetal tumors account for $31 \%$ of hepatoblastomas and embryonal tumors account for $19 \%$ of hepatoblastomas (5).

The hepatoblastoma treatment is relatively standardized, the only controversy matter between the European and American study groups remains the time of the surgical intervention. The International Childhood Liver Tumors Strategy Group (SIOPEL) recommends pre-surgical chemotherapy followed by tumor excision and then a short period of post-surgical chemotherapy (6). The American study group recommends surgical intervention at the moment of diagnosis (applicable in $50 \%$ of patients) followed by post-surgical chemotherapy (7).

We report a case of hepatoblastoma with an unfavourable evolution and highlight the importance of liver transplantation for the management of such cases.

Informed written consent was obtained from the patient's mother before the publication of this case report.

\section{PATIENT PRESENTATION}

\section{Presenting concerns}

A two-year old male patient was admitted to our clinic for the investigation of an abdominal mass. The patient did not have any significant personal or family medical history prior to presentation.

\section{Clinical findings}

Physical examination on admission revealed an influenced general state, constitutional growth delay (Weight $=7.9 \mathrm{~kg}<3^{\text {rd }}$ percentile, Height $=75 \mathrm{~cm}<3^{\text {rd }}$ percentile), marked skin pallor. The abdomen was painful, distended, with a firm mass in the right flank.

\section{Diagnostic focus and assessment}

Blood results showed anemia (Hgb: $9.5 \mathrm{~g} / \mathrm{dl}$ ), leukocytosis $\left(24,900 / \mathrm{mm}^{3}\right)$, neutrophilia $(16,000 /$ $\left.\mathrm{mm}^{3}\right)$, thrombocytosis $\left(994,000 / \mathrm{mm}^{3}\right)$, abnormal liver tests (AST- 240 U/1, ALT- within normal limits), elevated LDH (618 U/1) and high inflammatory markers (CRP- $104.56 \mathrm{mg} / \mathrm{dl}$, ESR- $65 \mathrm{~mm} / \mathrm{h}$ ).

Abdominal ultrasound showed a large, solid hepatic tumor. A CT scan was performed, which described a large 59/69/55 $\mathrm{mm}$ tumor located in segment 6 with heterogeneous structure and areas of necrosis, adherent to the external oblique muscle and with thrombosis of the right portal vein, no pulmonary metastatic lesions, horseshoe kidneys (Fig. 1).

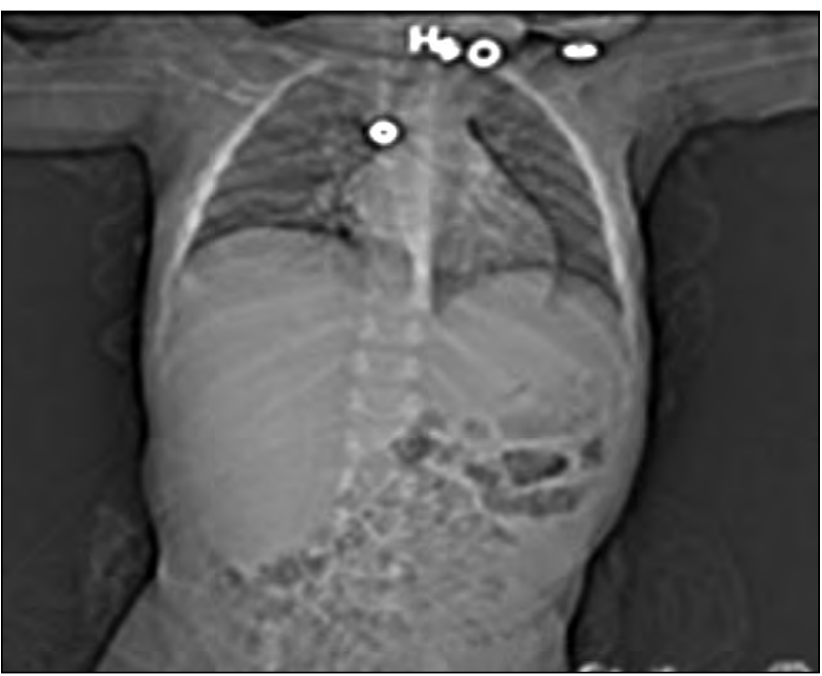

FIGURE 1. Abdominal CT scan showing large hepatic tumor

We excluded an infection with hepatitis B, C, HIV, Toxoplasma gondii, Epstein-Barr virus or citomegalovirus. Vanil-mandelic levels in the urine were determined in order to exclude the diagnosis of neuroblastoma, with a normal value: $1.11 \mathrm{mg} / 24 \mathrm{~h}$.

AFP levels were highly elevated - $174200 \mathrm{UI} / \mathrm{ml}$ (Normal: 0.92-6.73).

Taking into account the patient's age, elevated AFP levels, thrombocytosis, and imaging data the suspicion of a hepatoblastoma was raised. A biopsy was performed and the histopathological examination showed a histological aspect of an embryonic epithelial hepatoblastoma (Fig. 2).

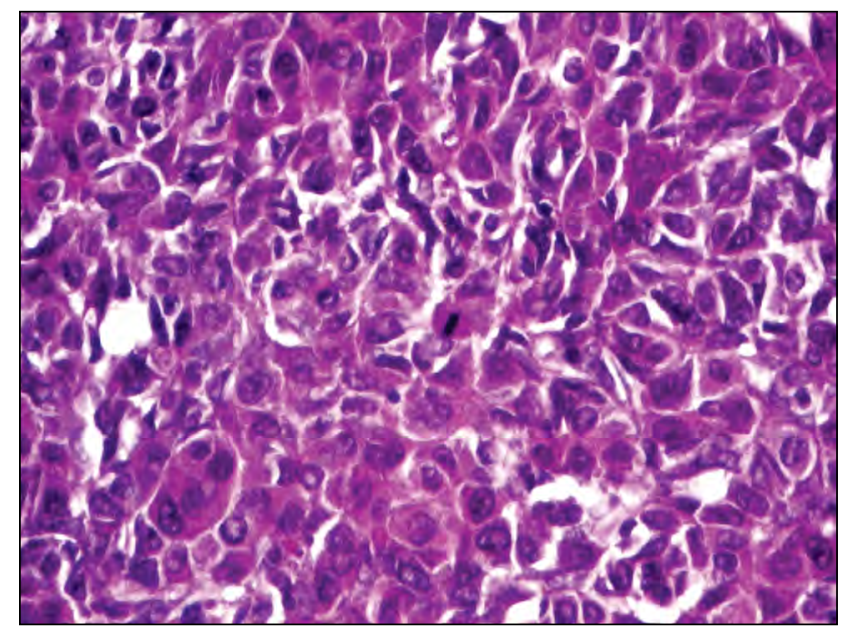

FIGURE 2. Hepatoblastoma. Hematoxilin-eosin

\section{Therapeutic focus and assessment}

Once the diagnosis of neuroblastoma - High Risk (due to the portal vein involvement) was con- 
firmed, chemotherapy was initiated, according to the International Childhood Liver Tumors Strategy Group 3HR trial, which involved 4 cycles of Cisplatin and 4 cycles of Carboplatin-Doxorubicin. Following chemotherapy the patient developed medullary aplasia requiring blood transfusion and granulocyte colony-stimulating factors.

AFP values decreased to $29.9 \mathrm{ng} / \mathrm{ml}$ and the abdominal CT scan showed a small reduction in tumor size, $60 / 32 / 54 \mathrm{~mm}$.

First surgery involved right portal branch ligation, followed by a cycle of Cisplatin. Step two of the surgical procedure (tumor resection) was performed five months later followed by a final cycle of Cisplatin.

\section{Follow-up and outcome}

Six months after the second surgery a relapse was suspected due to hepatomegaly, confirmed by very high levels of alpha-feto protein $(56,8163 \mathrm{UI} /$ $\mathrm{ml}$ ) and abdominal CT scan which showed multiple lesions in the right and left liver lobes. Chemotherapy was resumed, with 3 cycles of CarboplatinDoxorubicin and 4 cycles of Cisplatin/Vincristin/5Fluorouracil. A bone scintigraphy and a thoracic CT scan were performed in order to exclude bone or pulmonary metastases, both negative.

Following the guidelines for relapses after surgery and chemotherapy, a liver orthotopic transplantation with segments 2-3 from a living related donor was performed. Postoperative evolution of the graft was favorable and the patient is diseasefree for three years.

In order to evaluate the degree of fibrosis of the transplanted liver we performed liver elastography and Fibrotest ${ }^{\circledR}$, both of the investigations were in normal range,

\section{DISCUSSION}

A large number of congenital syndromes and malformations have been described in patients with hepatoblastoma, such as Beckwith-Wiedemann syndrome, familial adenomatous polyposis and renal malformations. It was found to be more frequent in low-birth weight and premature infants $(8,9)$. Our patient did not have any of the abovementioned clinico-pathological risk factors associated with higher incidence of hepatoblastoma. In terms of possible associated conditions, he presented horseshoe kidneys with hypoplasia of the right kidney. Chan et al. proposed a new association be- tween hepatoblastoma and hypoplastic kidneys, in a retrospective study performed in the Los Angeles Childhood Oncology Department and reported three such cases (10).

Hepatoblastoma prognosis depends on the tumor extension at the moment of diagnosis, on its excisability and on the histological type $(11,12)$. In our case, the involvement of the portal vein and the large dimensions of the tumor led to the inclusion of the case in stage III, unresectable neuroblastoma. In terms of histology, pure fetal type confers the best prognosis (13). Our patient's tumor presented an epithelial, embryonal and fetal mixed histology, with a less favorable prognosis.

Hepatoblastoma treatment combines preoperative chemotherapy with surgical excision, allowing a survival rate of over $70 \%$ (14). Complete surgical resection is the cornerstone of treatment; however less than $40 \%$ of patients have resectable disease at diagnosis (15). If the tumor is considered unresectable and the patient is at high risk for complications, a biopsy is taken and preresection chemotherapy is indicated (5).

Asmar and Rassi described the case of a 8 months old female infant with a mixed mesenchimal and epithelial type hepatoblastoma who did not respond to chemotherapy, but was treated succesfully via surgical resection, with no recurrent disease after 15 years (16).

Hepatoblastoma is considered to be non-surgical when: the tumor is extremely large, involving the risk of severe hemorrhage; both hepatic lobes are affected, the hepatic vein or the inferior vena cava is involved (17). In our case we performed a two-step procedure: during the first step, the right portal vein was ligated. This maneuver produced fibrosis of the right hepatic lobe and an enlargement of the left hepatic lobe. In these conditions, during the second step, a resection of the tumor in fibrous tissue was easier to perform and the risk of hemorrhage was decreased. This technique was first described in a pediatric patient by Chan et al., who performed a liver partition and portal vein ligation followed by tumoral resection in a patient aged 6 with a large hepatoblastoma. The patient had a favorable evolution, unlike our case who presented a relapse 6 months after surgery (18). Another study by Qazi et al. described an early tumoral reccurence in an infant with hepatoblastoma, who developed a relapse within the first month after the two-step surgery (portal vein ligation and subsequent tumoral resection) (19). 
Despite the aggressive chemotherapy regimen and the complete resection of the tumor, our patient presented a recurrence 6 months after surgery, with multiple lesions in the right and left liver lobes. A "salvage" liver transplantation was the only viable option for the patient. A liver orthotopic transplantation with segments 2-3 from a living related donor (the mother) was performed, with favorable post-operative and long-term evolution. Fernandez et al. reported a case of a chemoresistant hepatoblastoma in a patient with trisomy 18 , who developed recurrent disease after surgery and aggressive chemotherapy with cisplatin, vincristine, and 5 fluorouracil. Similar to our case, the patient underwent rescue orthotopic liver transplantation with a good long-term outcome (20).

Primary liver transplantation is indicated in the case of multifocal or solitary hepatoblastomas invading all four liver sectors or in centrally located tumors with close proximity to the major veins. Liver transplantation appears to have a better outcome when performed as a primary procedure rather than a salvage procedure for recurrent disease. Otte et al. reported $85 \%$ survival for 7 children un- dergoing primary liver transplantation and only $40 \%$ for 5 children who underwent "rescue" transplantation (21).

Liver elastography can be used to non-invasively detect the development of fibrosis in the transplanted liver, with good accuracy (22). We performed shear-wave elastography in order to evaluate the degree of liver fibrosis in our patient and compared it with the result of Fibrotest ${ }^{\circledR}$ (an algorithm developed by Biopredictive as an alternative to liver biopsy, which uses the serum levels of alpha-2 macroglobulin, haptoglobin, apolipoprotein A1, total bilirubin, gamma-glutamiltranspeptidase). Both of the investigations were in normal range, indicating no liver fibrosis.

\section{CONCLUSION}

Hepatoblastoma is a very rare pathology in children. Elevated AFP and thrombocyte levels point to the diagnosis of hepatoblastoma. For patients with extensive, non-resectable tumors or disease relapse after chemotherapy and surgery, liver transplantation remains the final, salvaging option.

\section{Conflict of interest: none declared Financial support: none declared}

\section{REFERENCES}

1. Zhang J, Xu F, Chen $\mathrm{K}$ et al. An effective approach for treating unresectable hepatoblastoma in infants and children: Pre-operative transcatheter arterial chemoembolization. Oncol Lett. 2013; 6(3): 850- 854

2. Khaderi S, Guiteau J, Cotton RT et al. Role of liver transplantation in the management of hepatoblastoma in pediatric population. World $\mathrm{J}$ Transplant. 2014; 24; 4(4): 294- 298.

3. Perilongo G, Shafford EA. Liver tumors. Eur J Cancer. 1999; 35: 953-959.

4. Schnater JM, Kohler SE, Lamers WH et al. Where do we stand with hepatoblastoma? A review. Cancer. 2003; 98: 668-678.

5. Finegold MJ, Lopez-Terrada DH, Bowen J et al. Protocol for the examination of specimens from pediatric patients with hepatoblastoma. Arch Pathol Lab Med. 2007; 131: 520-529.

6. Perilongo G, Shafford E, Maibach R et al. International Society of Paediatric Oncology - SIOPEL 2. Risk-adapted treatment for childhood hepatoblastoma; final report of the second study of the International Society of Paediatric Oncology - SIOPEL 2. Eur J Cancer. 2004; 40: 411-421.

7. Ortega JA, Douglass EC, Feusner JH et al. Randomized comparison of cisplatin/ vincristine/5-fluorouracil and cisplatin/continuous infusion Doxorubicin for the treatment of pediatric hepatoblastoma (HB): A report from the Children's Cancer Group and the Pediatric Oncology Group. J Clin Oncol. 2000; 18: 2665-2675.

8. Von Schweinitz D. Hepatoblastoma: Recent developments in research and treatment. Seminars in Pediatric Surgery. 2012;21:21-30.

9. Paquette $\mathrm{K}$, Coltin H, Boivin A et al. Cancer risk in children and young adults born preterm: A systematic review and meta-analysis. PloS One. 2019; 14(1): e0210366.
10. Chan R, Mascarenhas L, Venkatramani R. Hepatoblastoma and hypoplastic kidneys: a new association. Pediatr Blood Cancer. 2014;61(8):1476-8.

11. Stocker JT. Hepatic tumor in children. In: Suchy FJ, Sokol RJ, Balistreri WF, editors. Liver disease in children. 2nd edition. Lippincott Williams \& Wilkins; 2001. 91: 5-49.

12. Herzog CE, Andrassy RJ, Eftekhari F. Childhood Cancers: Hepatoblastoma. The Oncologist. 2000;5:445-453.

13. Carceller A, Blanchard $\mathrm{H}$, Champagne $\mathrm{J}$ et al. Surgical resection and chemotherapy improve survival rate for patients with hepatoblastoma. J Pediatr Surg. 2001; 36: 755-759.

14. Ang JP; Heath JA, Donath S et al. Treatment outcomes for hepatoblastoma: an institution's experience over two decades. Pediatr Surg Int. 2007; 23(2):103-9.

15. Teran DA, Beltran OG, Bru RC et al. Efficacy of neoadjuvant therapy and surgical rescue for locally advanced hepatoblastomas: 10 year single-center experience and literature review. World J Gastroenterol. 2014; 20(29): 10137-10143.

16. Suk-Bae M, Hyun-Baek S, Jeong-Meen S et al. Hepatoblastoma: 15-year experience and role of surgical treatment. J Korean Surg Soc. 2011;81:134-140.

17. El Asmar A, El Rassi Z. Hepatoblastoma in childhood, long term survival achieved: 2 case reports and literature review. Int $J$ Surg Case Rep. 2016; 21: 55-58.

18. Chan A, Chung P, Poon R. Little girl who conquered the "ALPPS", World J Gastroenterol. 2014; 20(29): 10208-10211.

19. Qazi A, Syed A, Khan AW et al. Early multifocal recurrence of hepatoblastoma in the residual liver after $\mathrm{R} 0$ liver resection with ALPPS procedure: a case report. Ann Transl Med. 2016; 4(19): 375. 
20. Fernandez KS, Baum R, Fung B et al. Chemoresistant hepatoblastoma in a patient with mosaic trisomy 18 treated with orthotopic liver transplantation. 2011; Pediatric Blood \& Cancer 56(3):498-500.

21. Otte JB, Pritchard J, Aronson DC Brown J, Czauderna P, Maibach R et al. Liver transplantation for hepatoblastoma: results from the International Society of Pediatric Oncology (SIOP) study SIOPEL-1 and review of the world experience. Pediatr Blood Cancer 2004; 42:74-83.

22. Perry MT, Savjani N, Bluth El et al. Point Shear Wave Elastography in Assessment of Hepatic Fibrosis: Diagnostic Accuracy in Subjects With Native and Transplanted Livers Referred for Percutaneous Biopsy. Ultrasound Q. 2016; 32(3):201-7 